\title{
Piecewise 3D Printing of Crystallographic Data for Post-Printing Construction
}

\author{
Matthew L. Brown ${ }^{a, *}$, David Hartling ${ }^{b}$, Hamel N. Tailor ${ }^{a}$, Ken Van Wieren ${ }^{c}$, Gary B. Houghton ${ }^{d}$, \\ Ian G. McGregor ${ }^{d}$, Callum D. Hansen ${ }^{e}$, and Nabyl Merbouh ${ }^{a, *}$
}

\begin{abstract}
A method of 3D printing complex or challenging structures by breaking them into parts with connectors, printing each part separately, and then assembling the structure post-printing has been developed. This has advantages such as multicoloured printing, framework optimization and reduction, print time reduction, and can be used to bypass print tray size limits. This method is particularly applicable to extended structures such as coordination polymers, metal-organic frameworks, and hydrogen bonding networks, but examples where it can be used to simplify the printing of small molecules are also shown.
\end{abstract}

\section{Introduction}

$3 \mathrm{D}$ printing is a rapidly growing and increasingly versatile method of conveying chemical information, particularly useful in pedagogy. ${ }^{1-3}$ An admirable amount of effort from the chemical and crystallographic communities has rapidly advanced the tools available, from complex multistep processes requiring multiple pieces of software ${ }^{3-5}$ to the ability to export crystallographic data to $3 \mathrm{D}$ printing files directly from common crystallographic software. ${ }^{6-8}$ This allows for the routine printing of many structures, ranging from small molecules ${ }^{6,7}$ through coordination polymers ${ }^{6}$ and complex proteins. ${ }^{8}$

We are demonstrating a proof of concept technique that will allow for crystallographic data to be printed in multiple parts, then assembled post-printing. This will allow for such benefits as multicoloured printing on monocoloured printers, bypassing the size restrictions of any 3D printer, reducing the amount of wasted framework (on printers where framework is required), making framework removal simpler (on printers where manual framework removal is required), and making printing more efficient via print tray optimizations. This method is somewhat more work intensive than printing a single unit, and relies on some proprietary CAD software, unlike the other published technique to print crystallographic data in multiple parts. ${ }^{9}$ However, that technique is currently limited to printing circular connectors, ${ }^{9}$ which we have found to be problematic due to the difficulty in preserving bond angles, and in printing curves on lower end printers. We are confident that if this method is interesting to the community, then support for it will be built into crystallographic software packages, or combined with existing methods. ${ }^{9}$ This will eliminate the need for these proprietary programs and make it readily more accessible, in the way tools for converting CIF files to printable file formats were created.

\footnotetext{
a Department of Chemistry, Simon Fraser University, 8888 University Drive, Burnaby, B.C., Canada. E-mail: matthew_brown_2@sfu.ca,nabyl_merbouh@sfu.ca

${ }^{b}$ Document Solutions, Simon Fraser University, 8888 University Drive, Burnaby, BC, V5A 1S6, Canada

${ }^{c}$ Science Technical Center, Simon Fraser University, 8888 University Drive,Burnaby, BC, V5A 1S6, Canada

${ }^{d}$ School of Engineering Science, Simon Fraser University, 8888 University Drive, Burnaby, B.C., V5A 1S6, Canada

$e^{e}$ Killarney Secondary School, 6454 Killarney St. Vancouver, BC V5S 2X7, Canada. Electronic Supplementary Information (ESI) containing further instructions, additional figures, the final version of all 3D printing files shown in this paper, and a set of video tutorials is available.
}

\section{Multicoloured Printing}

Many 3D printers are only capable of printing in one colour at a time. By dividing the structure up into subunits - atoms, moieties, symmetry, or any other criteria the user desires - and printing each subunit separately, any printer capable of printing multiple different colours of material can print a single structure with as many colours as the user has available. For example, the sodium chloride in Figure 1 was printed on a monocoloured 3D printer in two runs, and then assembled into one multicoloured structure. This could also be used to add different properties to different subunits, by using multiple printers that print different materials as shown in Figure 2.

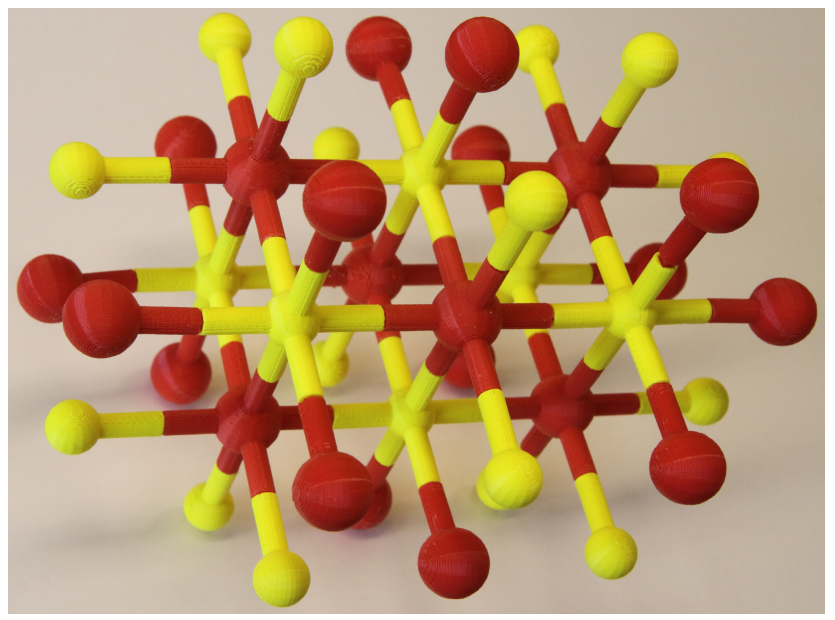

Fig. 1 This sodium chloride structure was printed in two print runs, using two different colours of filament on a monocoloured printer. Square pegs were used since each atom has fourfold rotational symmetry, making a connector that also has fourfold rotational symmetry both appropriate and convenient. ${ }^{10}$

\section{Bypassing Size Restrictions}

The vast majority of 3D printers are limited in what they can print by the size of the print tray in use. By printing the structure in multiple parts, each limited by the size of the print tray, these limits can be bypassed. For example, if an entire unit cell of the copper pyrazine structure shown in Figure 3 was printed as a simple unit at the same scale, it would be significantly larger then could be printed on the machine that produced the fragment shown in Figure 3. It was planned to demonstrate this by printing the whole unit cell in this manner, however due to budget constraints, 


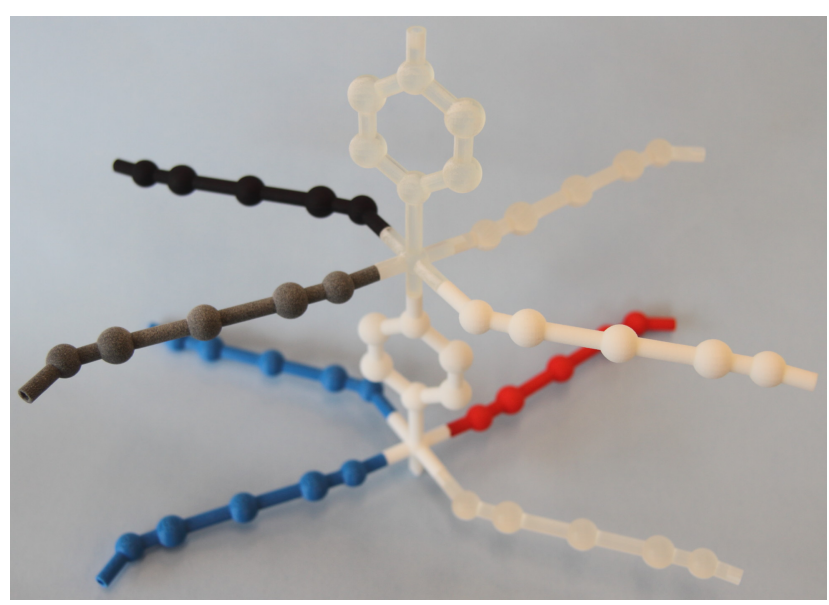

Fig. 2 This copper pyrazine was printed using Shapeways Versatile Plastic (white, red), Fine Detail Plastic (translucent), Professional Plastic (grey, black) as well as parts printed on Document Solutions Stratasys Fortus $250 \mathrm{mc}$ with ABSplus P430 (blue).This demonstrates how colours and materials can be combined in one model, how the output of multiple printings can be combined, and how shipping is simplified by splitting the part into multiple units, as this arrived from Shapeways with every part intact.

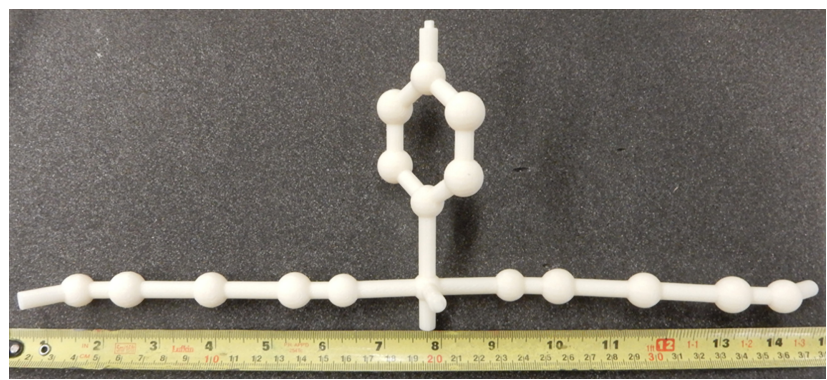

Fig. 3 A copper pyrazine dicyanoaurate coordination polymer ${ }^{11}$ repeat unit. Note that it lies flat on the backing foam because the bond on the obverse side of the metal centre is missing.

the structure was instead scaled down to that shown in Figure 2 as a proof of concept of the technique.

\section{Framework Waste and Removal}

Traditionally, three-dimensional printing has worked by moving the print head over the XY plane, laying down a layer of plastic as needed, then moving the printer a fixed step size in the $\mathrm{Z}$ direction and printing the next plane. This means that material that significantly overhangs the previous planes would need to be supported with scaffolding or framework. Many printers require the support framework to be broken off or cut away manually, which makes printing structures such as the sodium chloride (Figure 1) above difficult as the inner framework would have to be removed without damaging the surrounding structure. For this type of printer, the ability to print each subunit on its own with no or minimal framework required is a huge advantage as it will enable new types of structures to be printed. More advanced printers, such as the printer used to print the sodium chloride parts, can extrude a second type of polymer which can be dissolved away at the end of the print run. In this case, the mere fact that less framework can be used will not enable the printing of new types of structures. However, less framework material will need to be

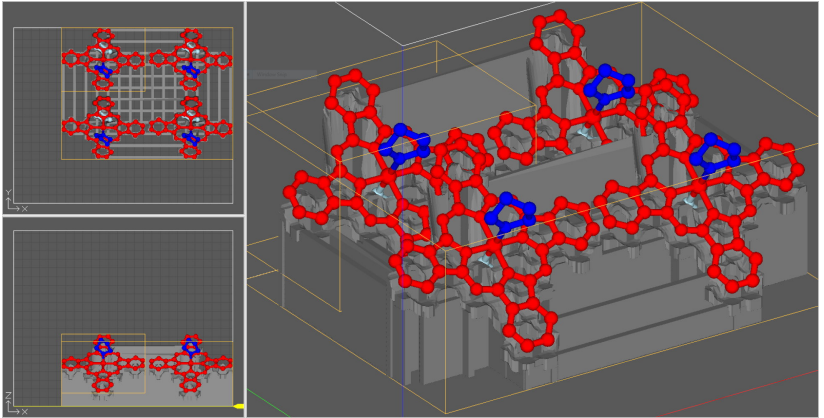

Fig. 4 Chromium phthalocyanine with two THF ligands printed as one unit. Note that more material would have to be used on the support framework than on the structure itself. ${ }^{12}$

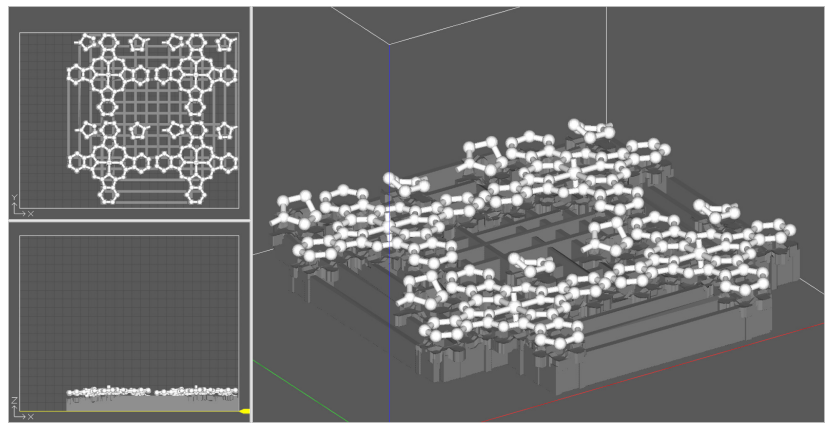

Fig. 5 Chromium phthalocyanine with two THF ligands split into three units. While in this case the print tray still imposes a limit of four units, significantly less support framework is needed. Additionally, more THF ligands or other structures can be printed in the spare room on the tray if desired. ${ }^{12}$

used which will save money and make the print run more ecologically friendly, and time not spent printing the framework is time spent printing the structure, making the print run substantially faster, and if commercial print services are being used, less expensive. An example of this is shown in Figures 4, 5 and 6. In Figure 4 the unit is printed as a single entity, and requires a very large amount of framework to support it. In Figure 5 the structure has been split into three parts: the chromium phthalocyanine and its two bound tetrahydrofuran (THF). This greatly reduces the amount of support framework needed. For very high end printers, the advantages of printing in subunits is more variable, though other aspects of the technique, such as bypassing size restrictions, multicolour printing, and print tray optimization are still applicable. This was tested when attempting to print a copper pyrazine dicyanoaurate coordination polymer (Figure 7) as a single unit (Figure 8). The print run took several days, and used several times the amount of material for the support framework as it did for the actual product.

\section{Print Tray Optimization and Misprint Mitigation}

For the majority of 3D printers, the layout of the structure on the print tray determines how much can be printed in a single run and how long the run will take. Therefore, printing subunits that can be optimally arranged on the print tray, instead of complete structures, provides a significant time efficiency, which reduces costs. For example, compare printing a full repeat unit of the copper pyrazine coordination polymer (Figure 7) to splitting this unit into a metal-pyrazine subunit and a dicyanoaurate subunit. 


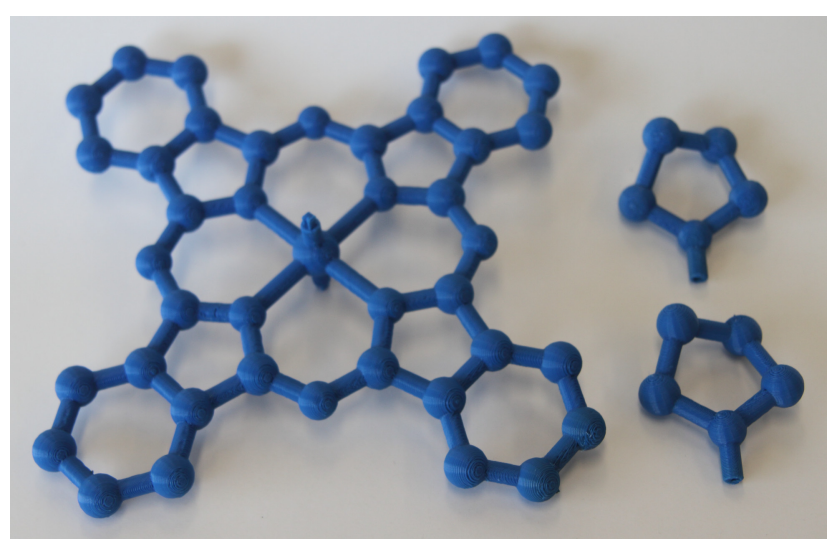

Fig. 6 Chromium phthalocyanine with two THF ligands printed as three units. ${ }^{12}$

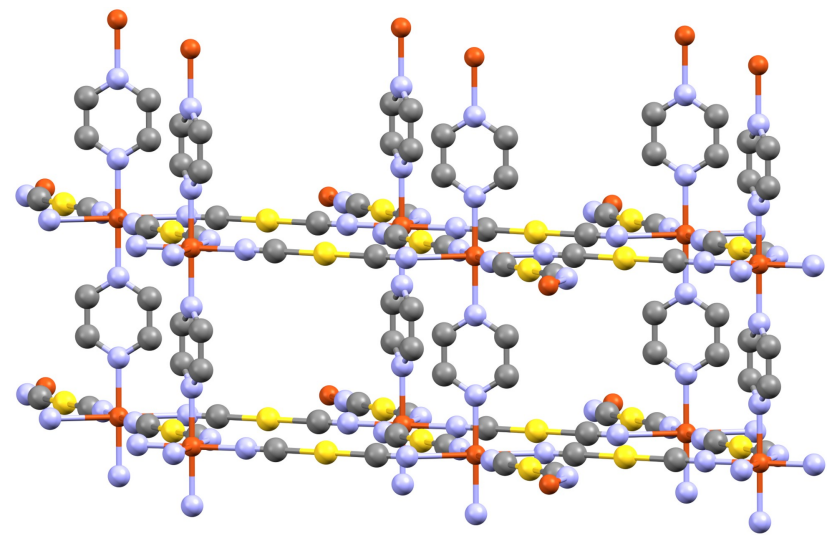

Fig. 7 A copper pyrazine dicyanoaurate coordination polymer that would require a very large print tray to print at a decent $\AA / \mathrm{cm}$ ratio due to the long, thin nature of the repeat units. ${ }^{11}$

Printed as a single $20 \mathrm{~cm}$ long unit (Figure 3) it takes up the entire print tray it was printed on, allowing only one repeat unit to be printed per run. If broken up into a subunit made of a metal centre and organic ligand, and a subunit comprised of the dicyanoaurate, multiple repeat units can be printed every run since they can be packed more efficiently on the print tray.

This also helps prevent waste if there is an error while printing. When this structure was printed, an error prevented one of the bonds from adhering to the copper in two successive print runs, as shown in Figure 3. This essentially ruined the entire print run, even though only one bond was misprinted. Had the structure been printed in smaller subunits, then only that subunit would have needed reprinting.

\section{Assorted Incidental Benefits}

In addition to the benefits this method of printing was designed to impart, additional benefits have been observed while undertaking the project. The first is that only the $\mathrm{RaF}_{2}$ structure (which has shallower connectors due to the angles involved), has needed adhesive to hold the structures together once assembled. This has allowed the careful disassembly of the structures, so that new parts can be added or they can be taken apart for transport. The authors would caution that this puts a lot of strain on the parts, and can lead to breakage and should not be undertaken on a regular basis, but it is possible to do without damage to the structure.

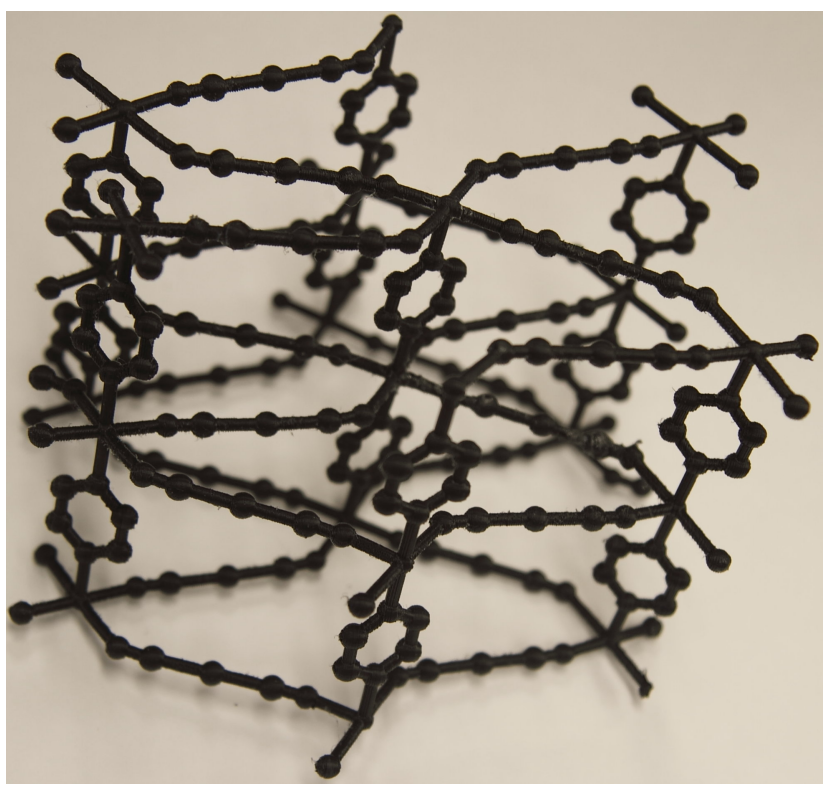

Fig. 8 This structure was printed as a solid object in one go. It is a failed attempt to print 7 as a single object.

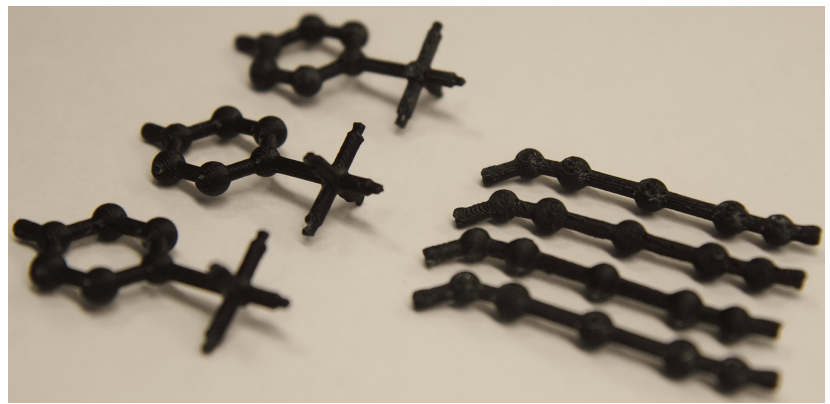

Fig. 9 Extremely small repeat units of the Copper Pyrazine Coordination Polymer Printed to the same scale as Figure 8

This also allows for structures to be repaired. If one part of an assembly is damaged, only that part needs to be reprinted and replaced, rather than the whole structure.

For example, in the printing of the structure shown in Figure 8 a failure in the temperature sensor caused warping in the structure of the bond angles of the layers after the first one were distorted leading to a ruined print run. If this had been printed in parts, this could have been corrected before the print run was complete and only the damaged parts replaced. To further exacerbate the problem this framework was knocked off a shelf and broke before a photo could be taken. It was repaired with glue for the purposes of the photo shown in 8 , a process that rendered the bond angles imperfect and took 45 minutes of careful work by the Simon Fraser University Technical Centre. It would have been much easier if the broken parts could simply be swapped out for new printed replacements like those shown in Figure 9.

An additional advantage of this method was noticed while transporting structures to a conference for display: Unassembled structures are much easier to safely transport, particularly if the final structure has large voids in it. Structures with large voids are difficult to transport, as only the outside can be easily padded. However, if rendered down into the component parts, it is easy to 
cut slits or hollows in packing foam or other material and safely transport them. Additionally, small objects are easier to arrange on the foam to minimize how much space they take up in a suitcase or package. This could have advantages if ordering parts from offsite printing institutions or travelling with structures.

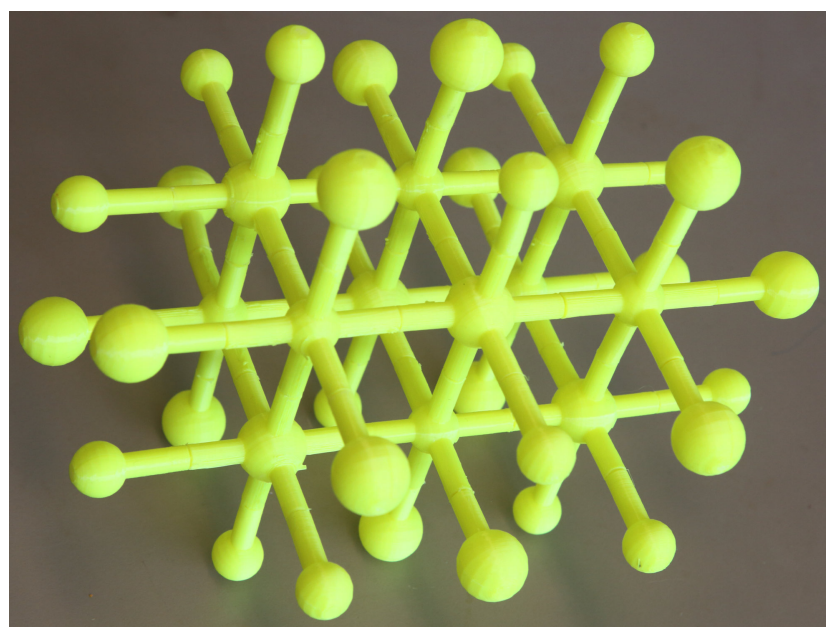

Fig. 10 This sodium chloride structure was printed on a Creality Ender 3, a sub$\$ 300$ hobbiest printer, by a secondary school student. This was done using the files from Figure 1.

Additionally, the peg and slot design and use of standard 3D printing files makes this technique quite usable on hobbyist or maker-space level printers. This was demonstrated by having a secondary school student print out and assemble the sodium chloride structure shown in Figure 10 on a sub-\$300CDN Creality Ender 3 printer.

\section{Method}

At its core this $3 \mathrm{D}$ printing technique is about splitting a structure into multiple parts and placing pegs and slots such that the two objects can be connected after they are independently printed. The first step in this process is taking the structure and dividing it into parts. This was accomplished using a combination of the Cambridge Crystallographic Data Centre's Mercury (multiple versions) and then either Materialise's Magics (v19.0.1.11) or the open source software MeshLab (64bit_fp v2016.12) from the Visual Computing Laboratory, the Istituto di Scienza e Tecnologie dell'Informazione and the Consiglio Nazionale delle Ricerche. ${ }^{13}$ First the structure was colour-coded in Mercury such that each piece to be independently printed was a different colour. This results in each moiety being a different colour and where two moieties intersect the connecting bond's colour will change at its midpoint as shown in Figure 1. The structure is then exported as a Virtual-Reality Markup Language file (*.wrl or VRML) as outlined previously. 6,8 This file was then imported into Magics or Meshlab where the Split Part by Color [sic] command (Magics) or a simple three step procedure outlined in the ESI (Meshlab) was used.

Each of the resulting parts was then saved as a separate STereoLithography (*.stl or STL) file. ${ }^{14}$ The structures were then reopened in Magics one at a time and then the repair functions were used to correct any errors created by Mercury's export function $^{15}$ and watertighten the structure, as outlined previously. 6,8 Once the parts were ready, they were imported into Dassault Sys- tèmes' SOLIDWORKS Educational Edition (Academic Year 20172018/2017 SP3.0) which was used to add the pegs and slots. Once the pegs and slots were added, each item was reimported into Magics to ensure no errors had been introduced. ${ }^{16}$

\section{Design of the Connectors}

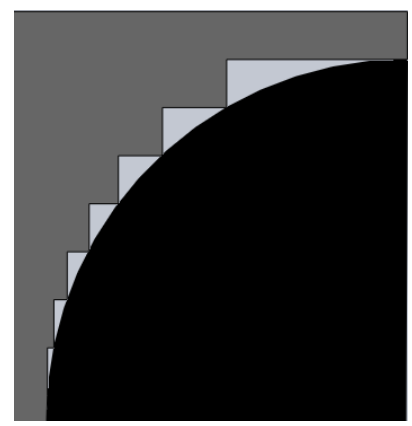

Fig. 11 An illustration showing why approximating a circular connector with rectangular layers is an issue. While it is an exaggeration, it is not much of one, as the number of layers used to approximate the circle is close in scale to that used to print bonds, as shown in Figures 12, 13, 15, 18, and 20.

While the concept of placing a slot in one half of a bond and a peg on the other half is simple, the execution has been more challenging than expected due to the nature of layered material $3 \mathrm{D}$ printing. One of the goals was to make it so that the pegs could be inserted into the slots only one way, such that it would be impossible to accidentally connect a ligand upside down, similar to how it is all but impossible to insert (all but the newest generation of) USB connectors backwards. ${ }^{17}$

Initial attempts using pegs made of merged circles or isosceles triangles were found to print unreliably. Circles were problematic due to the fact that the printer approximates the curve using rectangular layers as shown in Figure 11. The results of this printing attempt are shown in Figure 12.
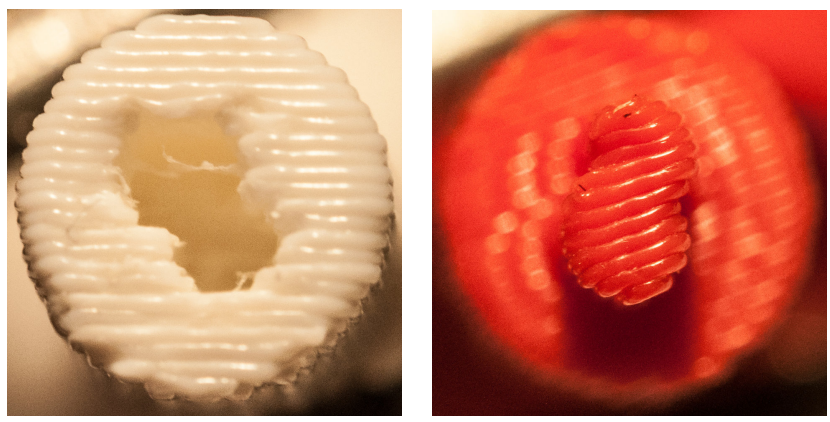

Fig. 12 The initial attempt was made using two differently sized overlapping circles. In addition to the problems approximating a circle with square layers it was found this generation of connectors was prone to breakage due to how thin the pegs were. Later iterations of the design greatly increased the amount of the surface area used to form the connectors.

Isosceles triangles were found to be unreliable as the vertices of the slot would fill in with material as in Figure 13.

Tests with square connectors were promising, but the initial batch of atoms for the sodium chloride structure (Figure 1) came back with a very high failure rate. The problem was quickly determined to be that if the slots and pegs were printed unaligned with 

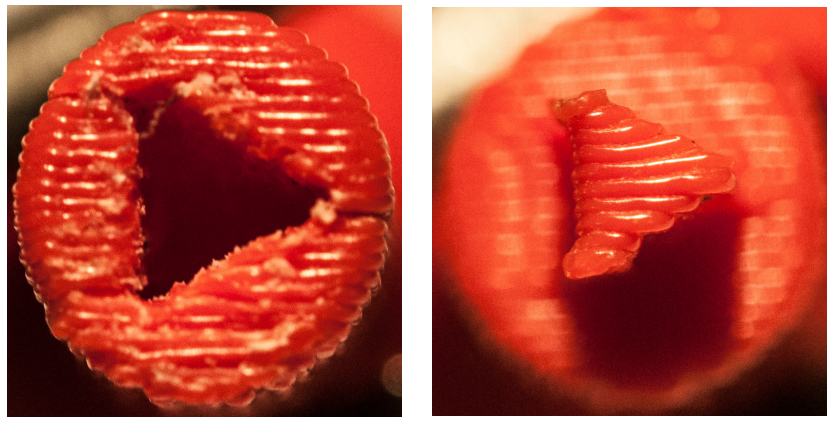

Fig. 13 The triangular connectors were far more successful, but the failure rate was quite high and the quality of the fit was found to be inferior to the square connectors. Note that the peg shown on the right is an earlier prototype then the slot on the left, thus the smaller size.

the direction of print, deformation of the connectors occurred. Without changing the $3 \mathrm{D}$ printing files (that is, printing the same structure from the same data), the structure was printed again, but with the connectors aligned to the direction of print, as illustrated in Figure 14. This necessitated the use of more framework material, but resulted in every objected printed on that run being usable, otherwise there tends to be deformation in the sides of the square leading to many connectors that do not fit together, as shown in Figure 15. Square connectors were also discovered to be stronger than the isosceles triangular design due to the peg having twice the volume of material when the distance between the edge of the connector and the outer wall of the bond is kept constant.

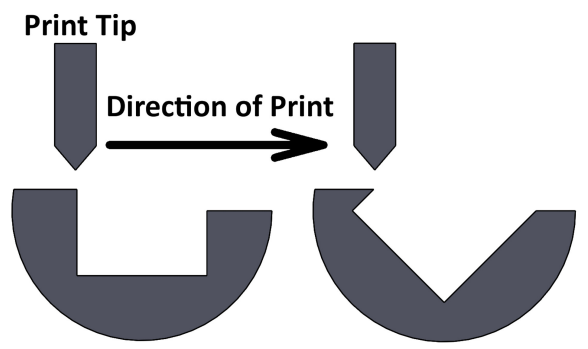

Fig. 14 Left: A connector aligned with the direction of print. Right: A connector not oriented with the direction of print.

The problem with square connectors is that squares have rotational symmetry, meaning there are four ways to connect a structure that uses square pegs. This is not always a problem, as shown in our sodium chloride structure (discussed below). Using square pegs is not an ideal solution, however, as in most cases this is undesirable as it creates opportunity for error during assembly, though it should be noted it introduces the opportunity for error during the addition of the connectors. Initial attempts at cutting off one to three corners of the square at a $45^{\circ}$ angle were promising, as demonstrated in Figure 16. However, using a non-right angle makes it impossible for all the sides to be aligned with the print direction, which was found to be a problem when printing the sodium chloride structure, discussed below.

The solution to this was to cut the appropriate number and combination of corners out of the square using smaller squares, as shown in Figure 17. This gives a large number of noninterchangeable connectors so that each bond can have a unique

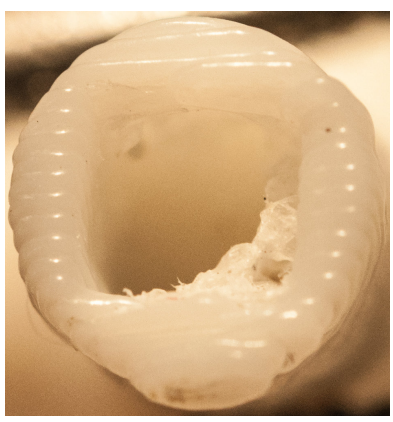

(a)

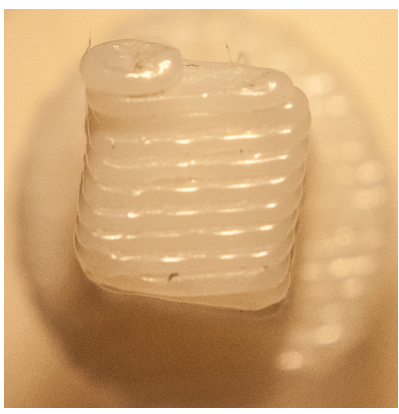

(c)

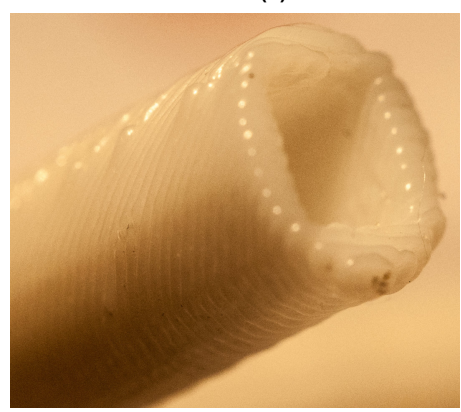

(e)

Fig. 15 a) A square slot printed non-aligned with the print layers. Note that despite the appearance of straight lines across the face of the bond, the layers are not actually aligned with the direction of print, as shown in e. b) A print job in which the square slot is perfectly aligned with the print layer. c) The pegs are more resilient to print direction, but errors do occur if the print direction is not aligned, as seen in this peg that was printed unaligned to the print direction. d) This peg was printed aligned to the print direction, giving it crisp lines except at the very bottom. This damage would not prevent a proper connection (and may be post-printing damage incurred while testing its ability to connect). e) A demonstration of how the lines in a) appear to be aligned but are not. The flaws in this peg are mostly not visible from this angle, but distortion can be seen at the top of the connector.

connector to avoid errors when assembling the structure. For example one corner can be removed to remove all two-dimensional rotational symmetry. Likewise, removing three corners will have the same effect. Additionally, if smaller sections of the square are removed many more possibilities are available, a small sample of which are shown in Figure 17. It should be noted that it is easy to create partially compatible connectors with this method. For example, in Figure 17, pegs using connector designs B and E both will fit into the slot for design A. Similar one-way compatibility exists between designs $\mathrm{C}$ and $\mathrm{D}$, and between all of $\mathrm{E}, \mathrm{F}$ and $\mathrm{G}$.

While these print errors may be specific to our style of polymer deposition 3D printer, the advantages of the rectangular connector design appear sufficient to warrant its use even on printers capable of printing arbitrary designs with perfect success rate. The 


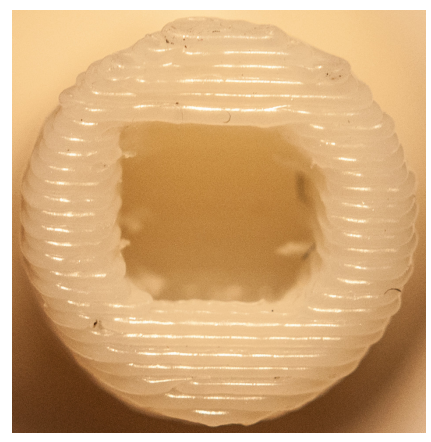

Fig. $16 \mathrm{~A}$ square slot with three of its corners cut off to remove rotational symmetry.
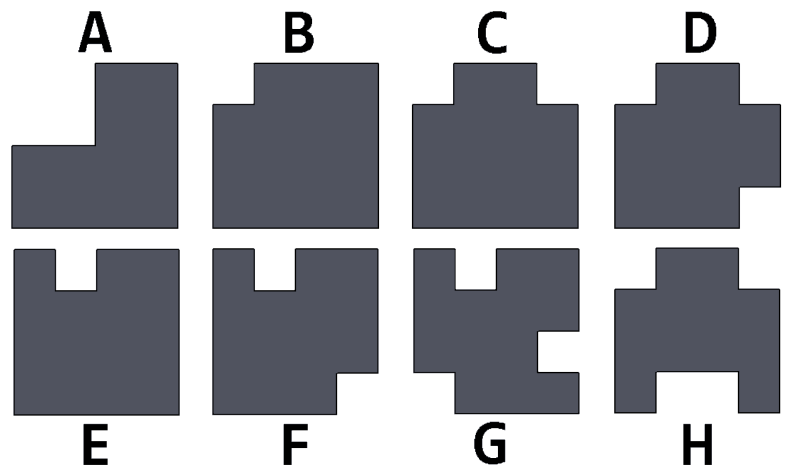

Fig. 17 A diagram illustrating some of the possible shapes that could be used for connectors by selective removal of squares from the overall shape. Note that the first two connectors would be partially compatible. Not all of these connectors have been tested by the authors.

rectangular design is strictly superior to the triangular design, due to the greater strength, and the ability to quickly produce many incompatible connectors that lack rotational symmetry. In theory designs made of overlapping circles would be viable on a higherresolution printer, and may even be stronger. The fact designs incorporating curves are limited to high end printers was felt to be too great a limitation to invest time studying their use as connectors given the successes of the rectangular connectors.

Printing structures that cannot be oriented to align the connectors to the print tray

In most structures, if the right choices are made in selecting the sub units all connectors can be aligned to the print tray. However, in some cases this is simply not possible, such as in lanthanide chemistry where geometries such as pentagonal prismatic are common, or in the chemistry of minerals where complicated packing mixtures lead to complex geometries. A technique was developed to deal with this: instead of separating the two print units at an angle perpendicular to the bond, they are separated at an angle perpendicular to the print tray. This results in connecters with oval cross-sections, as shown in Figure 20. This will likely necessitate the use of a spacer, shown in Figure 21, due to the fact the cut cannot easily be placed equidistant from both atoms. This technique was done entirely in Solidworks, without separating the file into parts in Magics or Meshlab first, though Mercury was still used to convert the CIF file to a STL or WRML file. The technique was tested using $\mathrm{RaF}_{2}$, which adopts the fluorite structure, due to

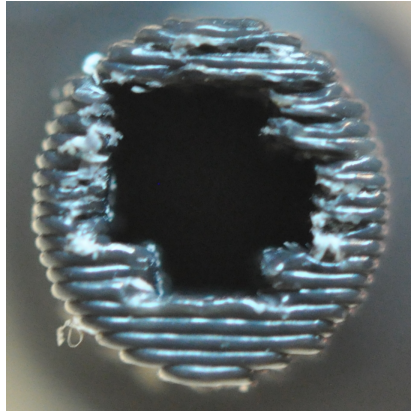

(a)

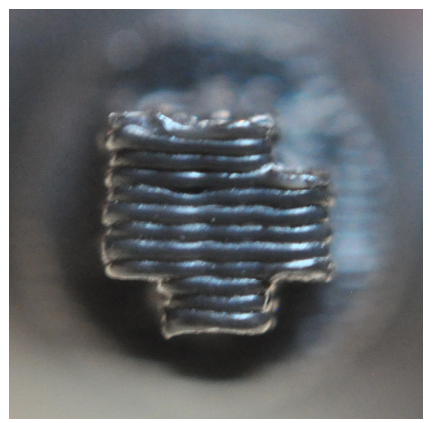

(b)

Fig. 18 The slot (a) and peg (b) of a correctly printed, working, connector with no rotational symmetry.

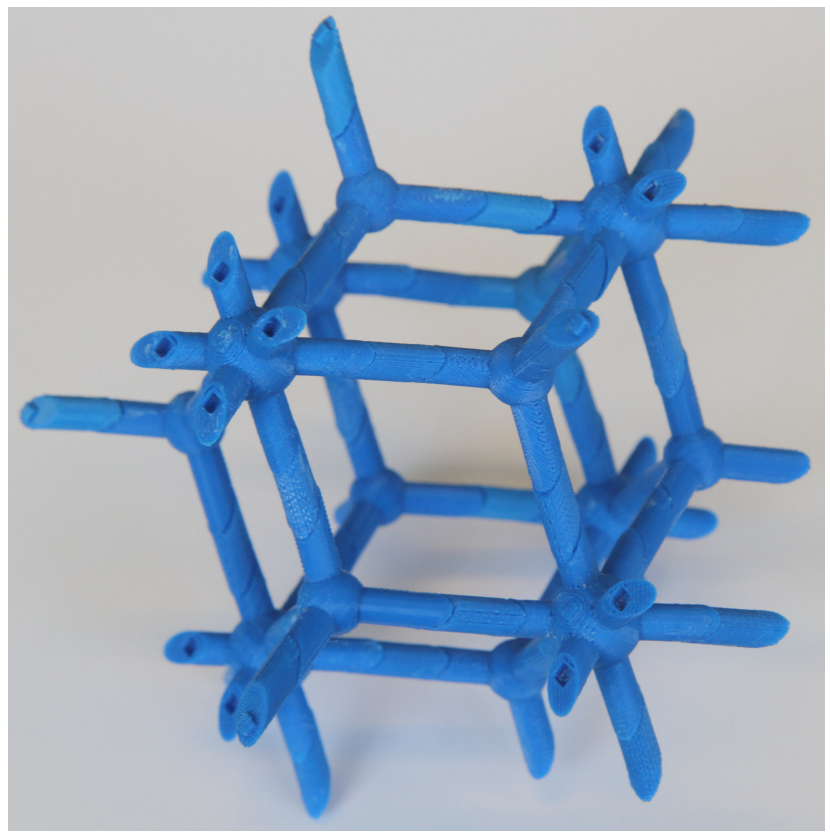

Fig. 19 This structure was constructed from parts printed on two printers. Instead of using the standard technique of separating the parts by colour using Mercury and either Magics or Meshlab, the structure was exported from Mercury and then sliced into pieces in Solidworks. This allowed the cuts to be made at angles nonperpendicular to the bonds, which allows the bonds to be oriented perpendicular to the print direction, despite the fact the atoms have tetrahedral and square prismatic geometries, which would normally preclude this.

its combination of a tetrahedral fluorine and square prismatic radium, as shown in Figure 19. This technique is rather more work than splitting the file based on colours, as the edits must be placed manually, the spacer cut at exactly the correct points, and it is difficult to place the connectors on identical places in the oval every time due to a lack of reference points and slight variations in the actual shape of the 'oval'. ${ }^{18}$ However, as shown in Figure 19 it was performed successfully. It should be noted that the complexity of this process is almost entirely due to using CAD software in a manner for which it was not intended, and a explanation of the technique and a video tutorial are both provided in the ESI. The actual operations themselves are quite simple, and could easily be performed in dedicated software on the files themselves, giving hope that automated tools for this task will be incorporated into standard crystallographic software in the near future. 


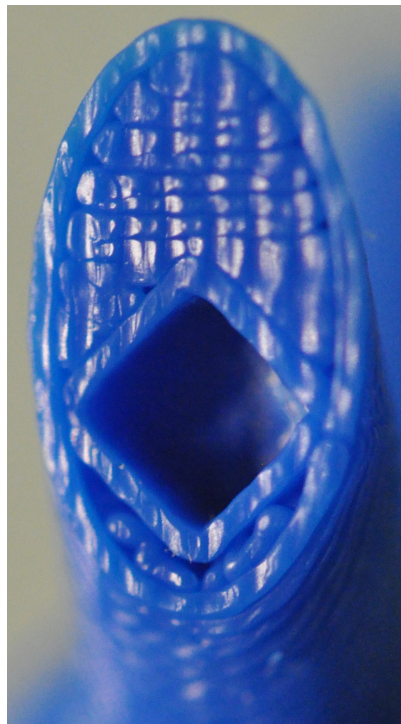

Fig. 20 Note the oval shape of the connector, and the fact the connector is not perpendicular to the bond.

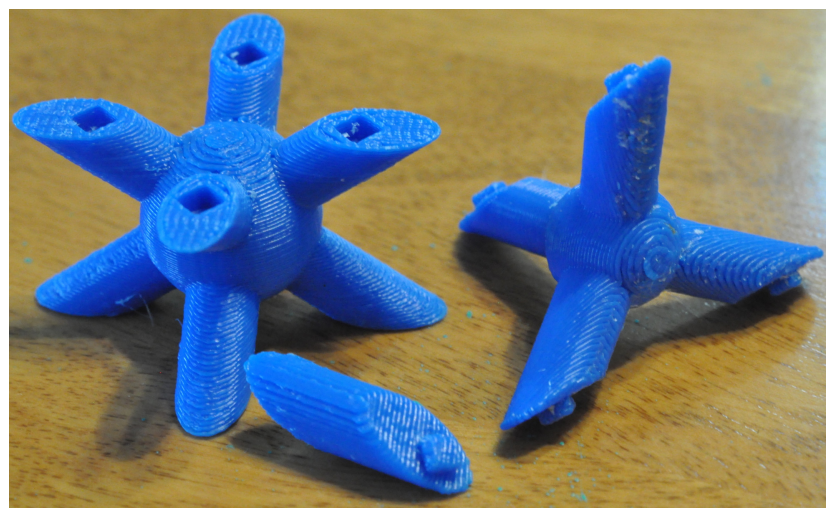

Fig. 21 The three parts needed to print the $\mathrm{RaF}_{2}$ structure. Left: radium atom with bonds, Centre: Spacer, Right: fluorine atom with bonds.

\section{Examples and Future Work}

Once initial prototyping was complete, square connectors were used to create the model of a sodium chloride crystal structure shown in Figure 1. Square pegs were chosen since they would allow full rotational freedom, as in each atom would not have a specified up or down. While there were initial issues with printing due to how the atoms were placed on the print tray, this structure was quite successful.

The next prototype was intended to demonstrate that it was possible to print a nontrivial extended structure, and as such a copper pyrazine dicyanoaurate coordination polymer ${ }^{11}$ was selected as the target. Initial tests were done attempting to create the entire repeat unit as one object, in an attempt to minimize the work required to turn a CIF file into a printable object. From this attempt it was learned that larger structures actually generate more work due to the additional complexity of aligning all of the pegs and slots. It was found to be challenging to keep track of every symmetry element needed for the whole structure at once. Determining the rotation and type of each connector was more challenging than adding more connectors, so only one moiety and its connections must be considered at a time. While not all users will find that to be a problem, particularly experienced crystallographers, the authors feel this additional challenge is worth noting, particularly given the much greater ease the authors had working these factors out after dividing the structure into two objects, necessitating the need for only one additional peg/slot pair. It was believed to be significantly easier to work out the appropriate rotations for the subunits shown in Figure 9 than to work out the rotations for the entire repeat units' connectors shown in Figure 3. After printing it was discovered that during this process, an error had been added to the print arrangement, resulting in an incorrect rotation of one of the dicyanoaurate units, as shown in Figure 22, an error that does not appear to be present in the initial attempt. It should be noted that if crystallographic software were to add this type of export as an option, the user would not have to work out these factors (since the software already knows what symmetry is present and can calculate how to place and align the connectors to preserve this), removing this element of difficulty from consideration. A technique and accompanying software implementation (Mol3DPrint) for adding connectors in an automated fashion was recently published, showing that this is possible. ${ }^{9}$ However, like our work, it relies on exporting the structure from the crystallographic software into a generic 3D file format, so the symmetry information available to dedicated crystallographic software is not available to Mol3DPrint. ${ }^{9}$ Therefore, we hope that the work done in this paper on removing rotational symmetry to preserve bond angle information, and the work in Mol3DPrint can be combined and incorporated into crystallographic software packages. This would allow those packages to add connectors based on the known symmetry of the crystal structure, and the rules established in this paper about connector orientation. Crystallographic software could output one file for each subunit, which could be defined either in a user specified fashion, or based on rules for minimizing the amount of framework required for the print job.

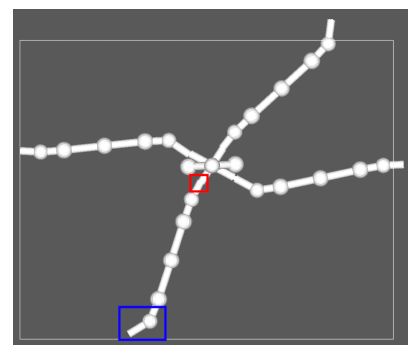

(a)

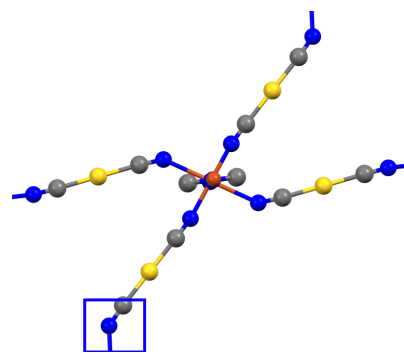

(b)
Fig. 22 An incorrectly rotated connector (highlighted in red) in causes a divergence between the physical model (Figure 22a) and the crystallographic data (Figure 22b). The divergence is highlighted in blue. ${ }^{19}$

This technique has been tested on multiple types of printers, including two Stratasys $250 \mathrm{mc}$ (Commercial-grade printers), an Ender 3 (A budget home-printer), and a series of plastics and steels printed from Shapeways (A complete list of prints is in the ESI), showing that it is device independent.

As noted in the section on Print Tray Optimization and Misprint Mitigation the original, large scale copper pyrazine dicyanoaurate failed twice due to a bond failing to adhere to the rest of the structure, as shown in Figure 3. After these failures the model was 
remade from scratch, separating the structure into two objects, one composing the metal centre and the organic ligand, and the other made of the dicyanoaurate moiety. This improved model also incorporated some of the newest generation of connectors that were under testing, shown in Figures 17 and 18. Not all of these connectors have been tested, but with the testing that has been done this series of shapes appear to print with a high success rate regardless of the quality of printer used.

\section{Conclusion}

While this procedure does involve multiple steps and quite a bit of manual work in the CAD software compared to recently published software, all the steps in this work could easily be automated and incorporated into existing crystallography software in the same way that exporting crystallographic data to 3D printing files has been. Printing crystallographic structures in multiple subunits and connecting them after printing allows for more efficient printing, enables some three-dimensional printers to print more challenging structures, allows the addition of multiple colours or the incorporation of multiple materials into a single structure, and grants the ability to print arbitrarily large extended structures. While this is not the first method of breaking structures into parts for printing, we feel that the detailed examination of the connectors used, and the improvements therein, will be useful to the community, both in improving existing software and in creating new methods for 3D printing crystallographic data. The increased reliability of printing connectors composed of rectangles, printed on a range of $3 \mathrm{D}$ printers at various price points, has been demonstrated. The adaptability of rectangular connectors has also been demonstrated, both for incompatible connectors to prevent human error during assembly, and for the removal of rotational symmetry. Removing rotational symmetry allows for more accurate bond angle preservation, which is an advantage over existing methods. We hope that this method attracts the attention of the crystallographic and chemical communities and is a valuable resource for those who are having trouble printing their structures, particularly those working in extended supramolecular structures.

\section{Conflicts of interest}

There are no conflicts to declare.

\section{Acknowledgements}

The authors would like to thank the Natural Sciences and Engineering Research Council of Canada for its support via a Graduate Scholarship (MLB). The authors would also like to thank Document Solutions for contributing print time and materials. This work was supported by the Simon Fraser University Dean Of Science INSPIRE grant. Additional thanks to Alan A. Brown for photographic contributions, Christopher T. Brown for video editing, and to Mara H. Katz for help editing and proofreading.

\section{Notes and references}

1 S. Gražulis, A. A. Sarjeant, P. Moeck, J. Stone-Sundberg, T. J. Snyder, W. Kaminsky, A. G. Oliver, C. L. Stern, L. N. Dawe, D. A. Rychkov, E. A. Losev, E. V. Boldyreva, J. M. Tanski, J. Bernstein, W. M. Rabeh and K. A. Kantardjieff, J. App. Crystallog., 2015, 48, 1964-1975.
2 P. P. Rodenbough, W. B. Vanti and S.-W. Chan, J. Chem. Ed., 2015, 92, 1960-1962.

3 V. F. Scalfani and T. P. Vaid, J. Chem. Ed., 2014, 91, 11741180.

4 T.-H. Chen, S. Lee, A. H. Flood and O. Š. Miljanić, CrystEngComm, 2014, 16, 5488-5493.

5 P. J. Kitson, A. Macdonell, S. Tsuda, H. Zang, D.-L. Long and L. Cronin, Cryst. Growth Des., 2014, 14, 2720-2724.

6 M. L. Brown, K. Van Wieren, H. N. Tailor, D. Hartling, A. Jean and N. Merbouh, CrystEngComm, 2018, 20, 271-274.

7 P. A. Wood, A. A. Sarjeant, I. J. Bruno, C. F. Macrae, H. E. Maynard-Casely and M. Towler, CrystEngComm, 2017, 19, 690-698.

8 K. Van Wieren, H. N. Tailor, V. F. Scalfani and N. Merbouh, J. Chem. Ed., 2017, 94, 964-969.

9 P. J. Paukstelis, J. Chem. Ed., 2018, 95, 169-172.

10 P. Fontana, J. Schefer and D. Pettit, J. Cryst. Growth, 2011, 324, 207-211.

11 D. B. Leznoff, B.-Y. Xue, C. L. Stevens, A. Storr, R. C. Thompson and B. O. Patrick, Polyhedron, 2001, 20, 1247-1254.

12 W. Zhou, J. R. Thompson, C. C. Leznoff and D. B. Leznoff, Chem. Eur. J., 23, 2323-2331.

13 P. Cignoni, M. Callieri, M. Corsini, M. Dellepiane, F. Ganovelli and G. Ranzuglia, Eurographics Italian Chapter Conference, 2008. DOI: 10.2312/LocalChapterEvents/ItalChap/ ItalianChapConf2008/129-136.

14 STL files were used instead of VRML files due to a bug in this version of Magics that resulted in anything saved as a VRML file being shrunk by a factor of 100 .

15 Note that it is important to do this correction after separating the structure into parts due to how Mercury's export function works. When a bicolour bond is formed Mercury creates two separate cylinders that are each sealed. If the repair function is used on a multi-coloured structure it will remove the walls sealing this cylinder because they can't be seen from the outside. Likewise the two cylinders will not actually be physically connected when output from Mercury, but will become so when the repair function is used. This normally has to be repaired before printing a structure, but if the purpose is to separate the parts by colour this reduces the risk of damage from repairing, splitting, and re-repairing the structure.

16 If Magics is not used, other software such as Insight from Stratasys Ltd and ZEdit from Z Corporation can be used for the water-tightening and error checking. In some of our work Insight was also used to check the structures in addition to Magics.

17 The authors have observed this accomplished once. Neither the USB port in question nor the motherboard it was attached to survived.

18 Unfortunately, all the software involved converts everything to triangle meshes instead of using actual cylinders and spheres. This leads to variation in the actual shape of the oval even when the cuts are identical, leading to a lot of care having to be taken in locating the centre of the oval for use as a reference point. This sometimes happened in circles as well, but not to the same extent. 
19 Figure 22a was generated with 3DSystems 3DPrint Software Version 1.03. Using some software that allows the easy layout of your parts before printing is recommended, as to avoid this type of error. Using print tray layout software to check connector orientations was not thought up by the author until after the above parts had been printed. 